\title{
Dual Axis Self-tracking Solar Panel
}

\author{
Srijesh Chettri \\ Sikkim Manipal Institute of \\ Technology \\ SMIT, Rangpo Majitar \\ East Sikkim, India
}

\author{
Arpan Chettri \\ Sikkim Manipal Institute of \\ Technology \\ SMIT, Rangpo Majitar \\ East Sikkim, India
}

\author{
Susan Chettri \\ Sikkim Manipal Institute of \\ Technology \\ SMIT, Rangpo Majitar \\ East Sikkim, India
}

\begin{abstract}
Dual Axis Self tracking Solar Panel is a hardware model that can modify the azimuthal and the elevation angle to make the solar panel always perpendicular with the sun's rays to produce maximum output all day long. This paper helps in determining whether a controlled solar tracking system produce greater output voltage rather than a fixed solar panel.
\end{abstract}

\section{General Terms}

Azimuthal, elevation angle, tracking, solar, servo motor.

\section{Keywords}

PWM, LDR

\section{INTRODUCTION}

The increase in the world population has led to the increase in demand of the energy. The main source of energy comes from oil and coal which is the non-renewable form of energy which means that it will perish someday. Also due to these form of energy the nature is also being degraded as it contributes to the global warming leading to melting of ice caps and disrupting nature.

To overcome this problem of non-renewable source of energy we have in recent years start turning to towards the renewable form of energy. The renewable source of energy are solar, wind, tides, geothermal. Solar energy is radiant light and heat from the sun harnessed using a range of technologies such as photovoltaic, thermal electricity and etc. [1].

The solar cell or photovoltaic cell converts the solar rays into electricity by using the photovoltaic effect. The solar panel is composed of photovoltaic modules which are connected electrically and mounted on a structure. The majority of modules use wafer based crystalline silicon cells or thin-film cells based on cadmium telluride or silicon [1]. The electrical connections are made in series to achieve a desired output voltage and in parallel to give the desired current capability.

Arduino is a single board microcontroller which is a open source computing and development board [1]. It can be used to take inputs from various objects and be used to control many devices, sensors, motors, etc. it is less expensive and has extensible software and hardware.

\section{SOLAR TRACKER}

Sunlight has two components, the direct beam that carries about $90 \%$ of the solar energy, and the diffuse sunlight that carries the remainder [1]. The diffuse portion is the blue sky in the clear day and it increases proportionally on a cloudy day.

We know that the fixed solar panel only converts $30 \%$ of the total sun rays incident on it. So we need a solar tracker which moves according to the movement of sun and tries to maintain the rays always perpendicular to the panel thus giving out the maximum output.

This paper explains the design and working of a dual axis self -tracking solar panel. It uses servo motors, solar panel and arduino as a controller. The LDR (Light Dependent Resistor) provides the analog input to the controller which then converts it to the digital input. The movement of the solar panel is controlled by the output of a servomotor. A panel was easy enough to acquire, but the true work in the project was the design and fabrication of the balance of system and driving algorithm.

\section{METHODOLOGIES}

\subsection{Tracking the light/rays}

The LDR are placed at the same location i.e. above the solar panel, at minimum distance separated by two shadowers. Tracking the light/rays is done using a simple mechanism.



Fig 1: Location of LDRs and structure of shadower

The two vertical board like structure crossing each other at the center are known as shadower. When a light is falling on a LDR from a particular direction, the shadower partially shadows the adjacent LDR and fully shadows the LDR on the opposite end. This helps the microcontroller to know were the intensity of the light is more. Thus the microcontroller provides effective outputs to the servo motors for modifying the azimuthal and elevation angle. The microcontroller keeps on providing the required outputs to the motors until and unless the light falling on all the LDRs are more or less the same.

\subsection{Modifying the azimuthal and elevation angle}

Servo control from a microcontroller to the servos is done by sending each servo a PWM (Pulse width modulation signal), a series of repeating pulses of variable width [2]. The servo library allows an arduino board to control servo motors. Servos have integrated gears and a shaft that can be precisely controlled. The servo motor that we have used can allow the shaft to be positioned at various angles between 0 and 180 
degree. The arduino Uno board has 14 digital pins out of which six pins produce PWM signals which can be used to control servo motors. When a pulse of $1.5 \mathrm{~ms}$ width is provided to the servo motor, the servo motor will be at natural 90 degree position. Similarly, with a $1 \mathrm{~ms}$ pulse the servo will be at 0 degree, and with a 2 ms pulse, the servo will be at 180 degree. In this way we can obtain the full range of motion by updating the servo with a value in between [2].

The arduino uses the same mechanism of PWM to control the servo motors when it is required to modify the azimuthal or the elevation angle or in some cases both [2].

\subsection{Producing maximum output}

After the LDR sends control information (light intensity) to the arduino, according to this Intel the azimuthal and the elevation angle is modified using PWM signals. By how much degrees? The answer to this question lies in the fact that the solar panel has to be perpendicular to the solar rays in order to maximize the output and the initial position of the solar panel. The elevation and the azimuthal angle is incremented or decremented until and unless the light falling on all the LDRs are uniform. The uniformity of light falling on all the LDRs will in return make the solar panel perpendicular with the sun rays as the LDRs and the solar panel is lying on the same plane

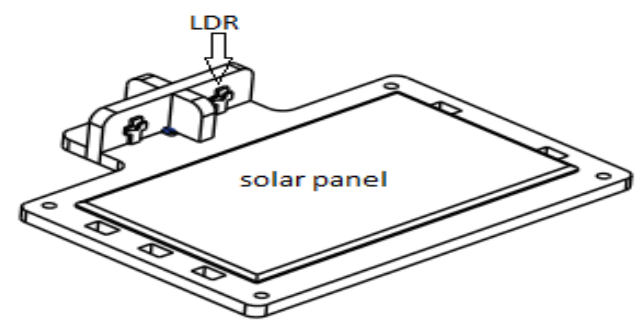

Fig 2: Solar panel and LDRs lying on the same plane.

SUN RAYS

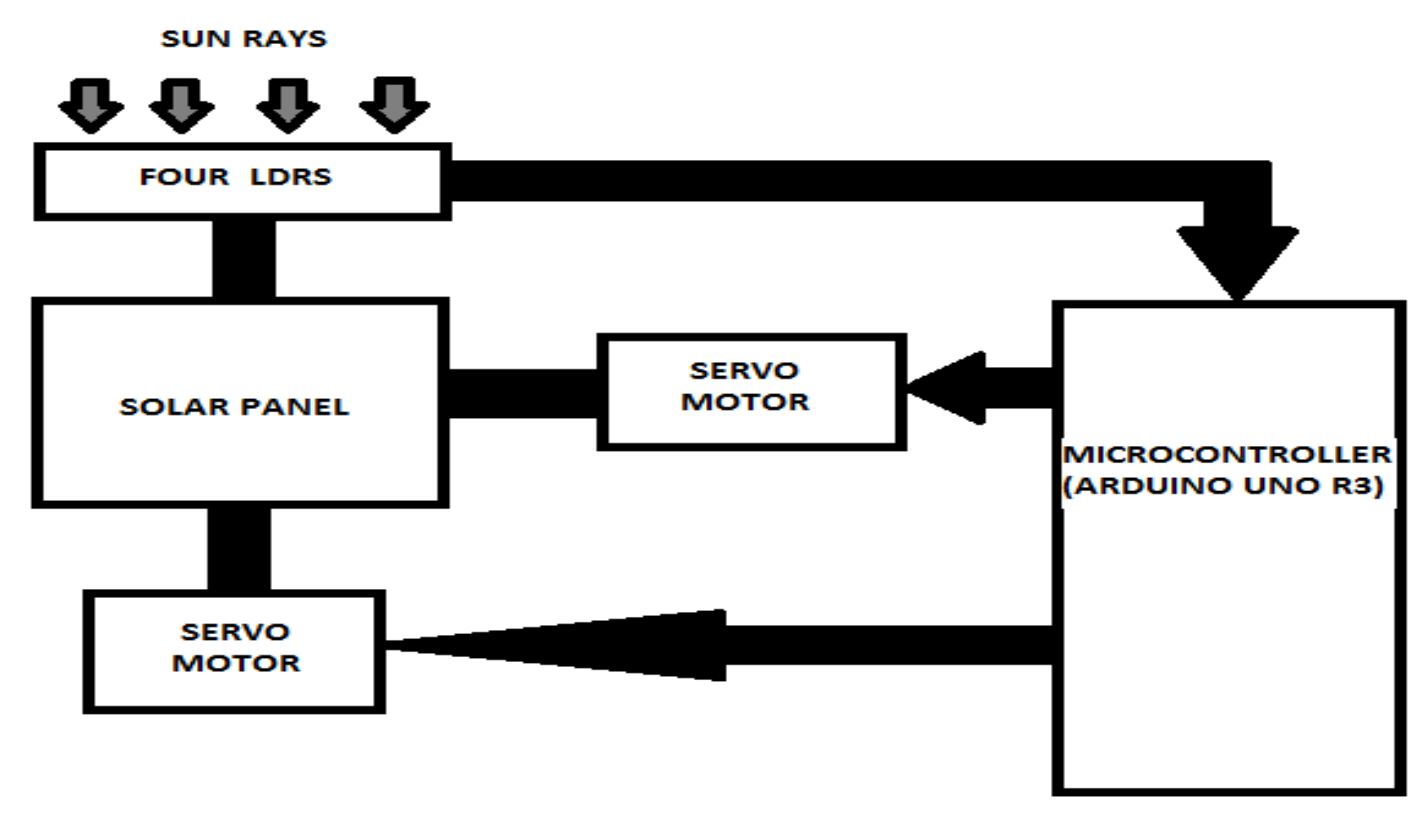

Fig 4: Block Diagram Of the Model

\section{CONNECTION DIAGRAM}

The LDRs are connected to A0, A1, A2 and A3 analog pins of the arduino board. Servo motors controlling the azimuthal angle is connecting to pin 9 and the servo motor controlling the elevation angle is connected to pin 10 respectively.

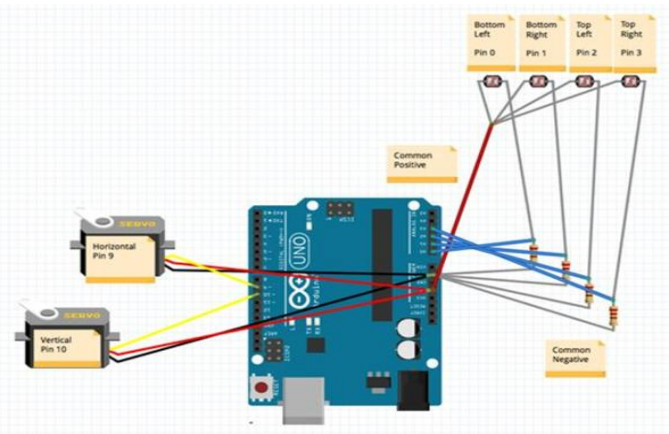

Fig 3: Connection diagram. 


\section{UNDERSTANDING THE CODE}

Initialize all the input pins for the LDRs, and output pins for the servo motors. Then store the values from the LDR to the arduino by defining four variables. Now we need to find the average between the two upper, bottoms, left and right LDRs. The next step is to find the difference between the averages of the top-bottom and also the difference between the averages of left-right LDR. The final value is required in figuring out the amount of modification that has to be made to the azimuthal and elevation angle.

\#include < Servo.h $>$ // include Servo library

Servo horizontal; // horizontal servo

int servoh $=180 ; / /$

int servohLimitHigh $=180$

int servohLimitLow $=0$;

Servo vertical; // vertical servo

int servov $=45$;

int servovLimitHigh $=80$;

int servovLimitLow $=0$;

// LDR pin connections

int ldrlt $=0$; //LDR top left - BOTTOM LEFT <--- BDG

int ldrrt = 1; //LDR top rigt - BOTTOM RIGHT

int ldrld = 2; //LDR down left - TOP LEFT

int ldrrd $=3$; //ldr down rigt - TOP RIGHT

void setup()

\{

Serial.begin(9600);

horizontal.attach(9);

vertical.attach(10);

horizontal.write(120);

vertical.write(45);

delay(3000);

\}

void loop()

int lt = analogRead(ldrlt); // top left

int $\mathrm{rt}=$ analogRead(ldrrt); // top right

int ld = analogRead(ldrld); // down left

int $\mathrm{rd}=$ analogRead(ldrrd); // down rigt

int dtime $=10$;

int tol $=50$;

int avt $=(\mathrm{lt}+\mathrm{rt}) / 2 ; / /$ average value top int avd $=(\mathrm{ld}+\mathrm{rd}) / 2 ; / /$ average value down

int avl $=(\mathrm{lt}+\mathrm{ld}) / 2 ; / /$ average value left

int avr $=(\mathrm{rt}+\mathrm{rd}) / 2 ; / /$ average value right

int dvert = avt - avd; // check the diffirence of up and down

int dhoriz $=$ avl - avr;// check the diffirence og left and right

if $(-1 *$ tol $>$ dvert $\|$ dvert $>$ tol) // check if the diffirence is in the tolerance else change vertical angle

\{

if (avt > avd)

servov $=++$ servov;

if (servov > servovLimitHigh)

\{

servov = servovLimitHigh;

\}

else if (avt $<$ avd)

\{

servov $=--$ servov;

if (servov < servovLimitLow)

servov = servovLimitLow;

vertical.write(servov);

\}

if $(-1 *$ tol $>$ dhoriz $\|$ dhoriz $>$ tol $) / /$ check if the diffirence is in the tolerance else change horizontal angle



else if (avl < avr) 


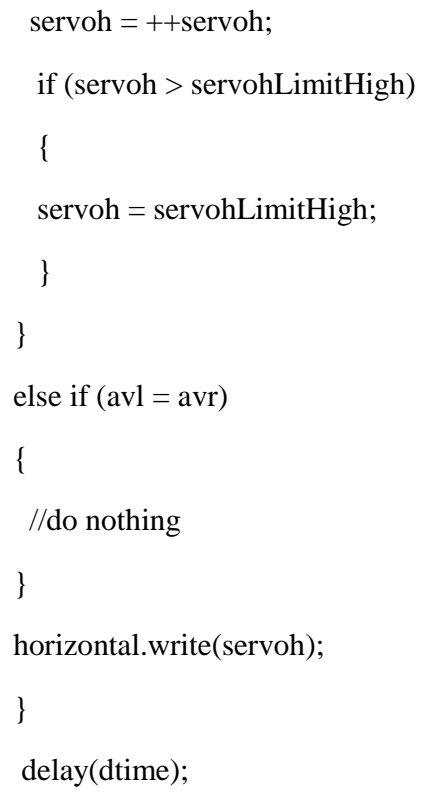

\section{FINAL MODEL}

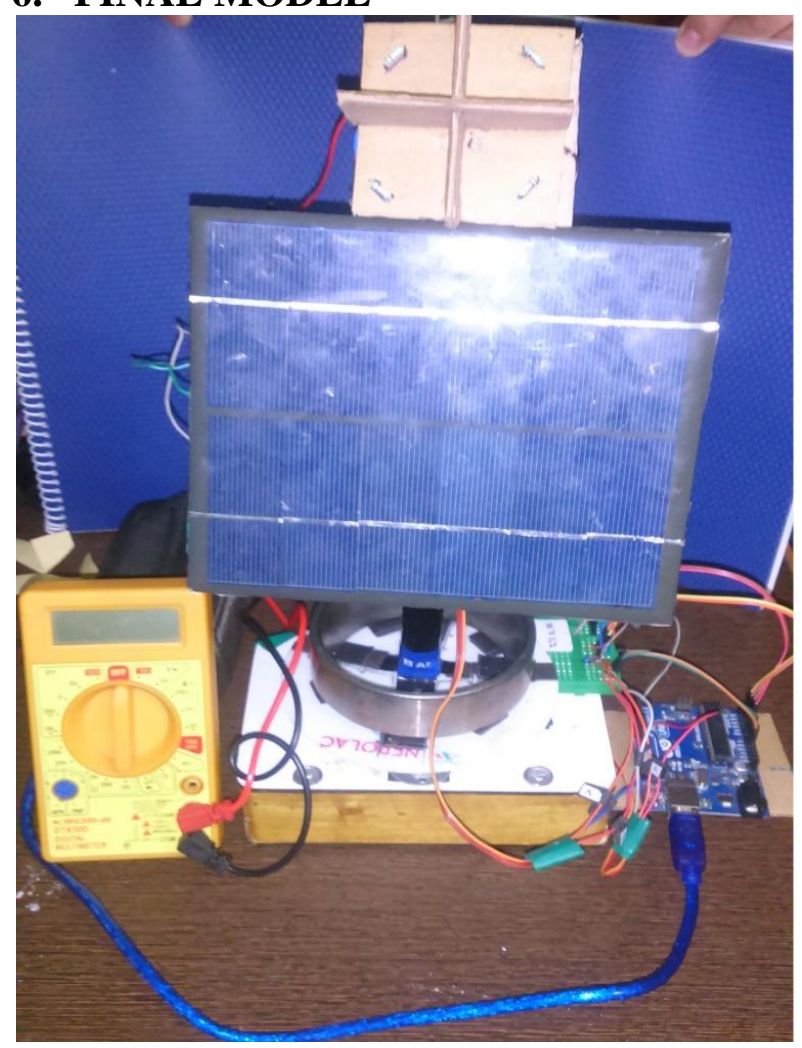

Fig 5: Image of the final model

\section{FUTURE SCOPE}

It can be synchronized with the Earth's rotation and revolution time to make the position of the solar panel more precise. The solar panel can be replaced with any other more effective solar devices to produce more output.

If this technology was to be employed on a larger scale many things would have to change. Instead of using small servo motors like we did, it is probable that larger DC motors with gearboxes or hydraulic systems would have to be utilized. As stated in the introduction, if we are to mitigate our influence on our environment by cutting down on fossil fuel usage, it is important to maximize energy output from clean energy sources, which makes them more competitive in the energy market. In terms of cost, once renewable sources become level with the dirty, outdated energy sources we will have a chance at moving away from smoggier skies, more intense storms, and more variance within seasons. Over the past decade, major research has been done and progress made in the solar cell field, but much of future innovation will need to come in the Balance of System field.

\section{CONCLUSION}

The hardware model for dual axis self- tracking solar panel has been made. We found out that the dual axis mechanism was lot better than the single axis as well as the stationery model. With the help of dual axis mechanism we could always keep the solar panel perpendicular with the sun rays thus getting maximum output.

\section{ACKNOWLEDGMENTS}

We are extremely grateful to our project guide Mr. Rochan Banstola (Associate Professor, E \& C Dept., SMIT) for insightful suggestions on the project work and for guiding us during the entire project with his encouragement, support and cooperation.

We would also like to extend our heart-felt thanks to our family and friends for their moral support, love and affection.

\section{REFERENCES}

[1] K P J Pradeep1, K Sai Prasad Reddy2,C Chandra Mouli3, K Nagabhushan Raju4, "Development of DualAxis Solar Tracking using Arduino with Lab VIEW", 1,3,4 Dept. of Instrumentation, Sri Krishnadevaraya University, Anantapur,Andhra Pradesh, India 2 Dept. of Electronics, Sri Krishnadevaraya University, Anantapur, Andhra Pradesh, India, International Journal of Engineering Trends and Technology (IJETT) Volume17 Number7-Nov2014

[2] www.learn.sparkfun.com

[3] Daniel spaizman, Dr. Tom Bensky, "An Investigation into Dual-Axis Solar Tracking", Faculty of the Physics Department California Polytechnic State University, San Luis Obispo, March, 2013.

[4] Saravanan C., Dr. M.A. Panneerselvam, I. William Christopher, "A Novel Low Cost Automatic Solar Tracking System", International Journal of Computer Applications (0975 - 8887) Volume 31- No.9, October 2011.

[5] www.wikipedia.com 